\title{
Guía de práctica clínica para el tratamiento farmacológico y psicológico de los pacientes adultos con un trastorno de ansiedad y un diagnóstico comórbido de trastorno por uso de sustancias
}

\author{
Clinical practice guideline on pharmacological and \\ psychological management of adult patients with an \\ anxiety disorder and comorbid substance use
}

\author{
Pilar A. Sáiz ${ }^{*}$, Gerardo Flórez ${ }^{* *}$, Manuel Arrojo***, Miquel Bernardo****, Ana González- \\ Pinto $* * * * *$, José Manuel Goikolea******, Iñaki Zorrilla*****, Ruth Cunill******, Xavier \\ Castells $* * * * * * *$, Elisardo Becoñ $* * * * * * * *$, Ana López*********, Marta Torrens*********, \\ Francina Fonseca $* * * * * * * * *$, Judit Tirado-Muñoz**********, Belén Arranz******, Marina \\ GARRIGA****, LUIS SAN******. \\ ${ }^{1}$ Estos autores han contribuido por igual a este trabajo. \\ * Universidad de Oviedo, Centro de Investigación Biomédica en Red de Salud Mental (CIBERSAM), Instituto de Investigación \\ Sanitaria del Principado de Asturias (ISPA), Servicio de Salud del Principado de Asturias (SESPA), Oviedo, España. \\ ** Unidad de Conductas Adictivas, Complejo Hospitalario de Ourense, CIBERSAM, Ourense, España. \\ *** Servicio de Psiquiatría, EOXI de Santiago de Compostela, España. \\ **** Hospital Clinic, Institut d'Investigacions Biomèdiques August Pi i Sunyer (IDIBAPS), Universitat de Barcelona, \\ CIBERSAM, España. \\ ***** Instituto de Investigación Sanitaria BIOARABA, OSI Araba, Hospital Universitario, CIBERSAM, UPV/EHU, Vitoria, \\ España. \\ ****** Parc Sanitari Sant Joan de Deu, CIBERSAM, Universitat de Barcelona, España. \\ ******* Grupo de investigación TransLab, Departamento de Ciencias Médicas, Universitat de Girona, España. \\ ******** Unidad de Tabaquismo y Trastornos Adictivos, Facultad de Psicología, Universidad de Santiago de Compostela, \\ Santiago de Compostela, España. \\ ********** Institut de Neuropsiquiatria i Adiccions (INAD), Parc de Salut Mar, RTA, Barcelona, España.
}

\section{Resumen}

Esta revisión resume las intervenciones farmacológicos y psicosociales que han sido llevadas a cabo en trastornos de ansiedad con un diagnóstico comórbido de trastorno por uso de sustancias y además proporciona recomendaciones clínicas respecto de cuáles elementos de intervención son útiles para hacer frente a los síntomas del uso de sustancias y los síntomas de ansiedad en pacientes con estas afecciones concurrentes. Se utilizó la mejor evidencia de ensayos controlados aleatorizados para evaluar las opciones de tratamiento. La fuerza de las recomendaciones se describió mediante el enfoque GRADE. Hay ensayos clínicos disponibles únicamente para el trastorno por estrés postraumático (TEPT) y para el trastorno de ansiedad. En cuanto al diagnóstico comórbido de trastorno por uso de sustancias, todos los estudios han incluido pacientes con consumo de alcohol, ninguno de ellos ha abordado el consumo de cocaína, cannabis o nicotina.

\begin{abstract}
This review synthesizes the pharmacological and psychosocial interventions that have been conducted in comorbid anxiety disorders and SUDs while also providing clinical recommendations about which intervention elements are helpful for addressing substance use versus anxiety symptoms in patients with these co-occurring conditions. The best evidence from randomized controlled trials was used to evaluate treatment options. The strength of recommendations was described using the GRADE approach. Clinical trials are only available for posttraumatic stress disorder (PTSD) and for social anxiety. Concerning the comorbid substance use, all the studies have included patients with alcohol use, none of them have dealt with cocaine, cannabis or nicotine use. Although some treatments have shown benefit for anxiety symptoms without benefits for alcohol or other substance use, only limited pharmacological approaches have been
\end{abstract}

Recibido: Abril 2020; Aceptado: Diciembre 2020.

Enviar correspondencia a:

Manuel Arrojo, MD. Hospital Psiquiátrico de Conxo. Plaza Martin Herrera 2, 15706, Santiago de Compostela, España.

E-mail: manuel.arrojo.romero@usc.es 
Aunque algunos tratamientos han mostrado beneficios para los síntomas de ansiedad sin beneficios para el consumo de alcohol $\mathrm{u}$ otras sustancias, solo se han ensayado enfoques farmacológicos limitados (sertralina, desipramina, paroxetina, buspirona, naltrexona y disulfiram). Nuestros resultados sugieren que 1) podemos (débilmente) recomendar el uso de desipramina sobre la paroxetina para aliviar los síntomas de ansiedad en pacientes con un TEPT y consumo de alcohol; 2) en estos pacientes, el uso de naltrexona para reducir los síntomas de ansiedad es también recomendable (fuerza débil); y 3) se pueden recomendar antidepresivos ISRS frente a placebo para reducir el consumo de alcohol (recomendación débil). Nuestra revisión pone de relieve la necesidad de realizar más investigaciones en esta área y de estudios más grandes, multisitio con muestras generalizables para proporcionar evidencia más definitiva para la práctica clínica.

Palabras clave: Ansiedad; trastorno por estrés postraumático; alcohol; inhibidores selectivos de la recaptación de serotonina; desipramina; naltrexona; disulfiram. assayed (sertraline, desipramine, paroxetine, buspirone, naltrexone and disulfiram). Our results suggest that 1) we can (weakly) recommend the use of desipramine over paroxetine to alleviate symptoms of anxiety in patients with a PTSD and alcohol use; 2) In these patients, the use of naltrexone to reduce symptoms of anxiety is also recommended (weak strength); and 3) SSRI antidepressants vs placebo can be recommended to reduce alcohol use (weak recommendation). Our review highlights the need for more research in this area and for larger, multisite studies with generalizable samples to provide more definite guidance for clinical practice.

Keywords: Anxiety; posttraumatic stress disorder; alcohol; selective serotonin reuptake inhibitors; desipramine; naltrexone; disulfiram.

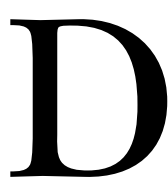
e acuerdo con la quinta edición del Manual diagnóstico y estadístico de los trastornos mentales (DSM5), los trastornos de ansiedad (AD) incluyen trastornos que comparten características de miedo y ansiedad excesivos y trastornos conductuales relacionados (American Psychiatric Association, 2013). Los AD se pueden clasificar según la causa del miedo: trastorno de ansiedad generalizada (situaciones cotidianas), trastorno obsesivo-compulsivo (pensamientos y conductas repetitivas), trastorno de pánico (ataques de pánico), trastorno por estrés postraumático (eventos traumáticos previos), trastorno de ansiedad social (juicios negativos por parte de otros) y fobias específicas (objetos o situaciones específi$\cos )$.

Los análisis de la encuesta epidemiológica centrada en la comorbilidad, la Encuesta Epidemiológica Nacional sobre Alcohol y Condiciones Relacionadas (NESARC; $N$ $=43.093$ ), ha revelado tasas sorprendentes de ansiedad y trastorno por abuso de sustancias concurrente (Compton, Thomas, Stinson y Grant, 2007; Hunt, Siegfried, Morley, Sitharthan y Cleary, 2013). Los AD aumentan la vulnerabilidad al abuso de sustancias (María-Ríos y Morrow, 2020; Vorspan, Mehtelli, Dupuy, Bloch y Lépine, 2015), siendo la tasa de prevalencia de por vida estimada en EE. UU. de $14,6 \%$ y las probabilidades de tener al menos un trastorno por uso de sustancias (TUS) de 1,7 (Smith y Book, 2008). Además, los consumidores de nicotina, alcohol y cannabis con un diagnóstico comórbido de trastorno de ansiedad mostraron un aumento del riesgo de transición a la dependencia (Lopez-Quintero et al., 2011). En cuanto al consumo de alcohol, los estudios clínicos han aportado evidencia tanto de la hipótesis de la automedicación, con efecto ansiolítico a corto plazo del alcohol como de efecto tóxico del consumo prolongado de alcohol que aumenta la ansiedad e induce síntomas de ansiedad, entre otros síntomas de abstinencia (María-Ríos y Morrow, 2020; Vorspan et al., 2015).

Los estudios han demostrado consistentemente que la concurrencia de TEPT y TUS aumenta la severidad de que cada condición y la dificultad del tratamiento (Clark, Masson, Delucchi, Hall y Sees, 2001). Los pacientes con comorbilidad de TEPT y TUS tienen un curso clínico más complejo y costoso en comparación con cualquiera de los trastornos por sí solos, incluido un funcionamiento de salud mental más deficiente, un aumento de los problemas crónicos de salud física, una peor adherencia y respuesta al tratamiento, más ingresos hospitalarios, más problemas interpersonales y tasas más altas de intentos de suicidio (Brady, Killeen, Brewerton y Lucerini, 2000; Driessen et al., 2008; McCauley, Killeen, Gros, Brady y Back, 2012).

En la práctica clínica, el manejo de un $\mathrm{AD}$ con un TUS se basa en el uso de estrategias de tratamiento efectivas para cada condición por separado (Ipser, Wilson, Akindipe, Sager y Stein, 2015; Sáiz et al., 2014). El tratamiento integrado pone el enfoque del tratamiento en dos o más afecciones simultáneamente y utiliza múltiples tratamientos, como la combinación de psicoterapia y farmacoterapia. La lógica del tratamiento integrado es que los enfoques múltiples son más integrales en el tratamiento de una afección que en realidad es una interacción de trastornos. Hasta la fecha, se ha reportado laa superioridad del enfoque integrado frente a tratamientos con enfoques individuales en pacientes con trastornos depresivos y uso de sustancias (Hesse, 2009). Sin embargo, el tratamiento 
psicoterapéutico basado en la evidencia para trastornos de ansiedad con un diagnóstico comórbido de trastorno por uso de sustancias no está respaldado empíricamente (Iqbal, Levin y Levin, 2019; Vorspan et al., 2015). Esta revisión resume las intervenciones farmacológicas y psicosociales que se han realizado en trastornos de ansiedad con un diagnóstico comórbido de trastorno por uso de sustancias y además proporciona recomendaciones clínicas respecto de cuáles elementos de intervención son útiles para hacer frente a los síntomas del uso de sustancias y los síntomas de ansiedad en pacientes con estas afecciones concurrentes.

\section{Métodos}

\section{Formulación de preguntas clínicas}

De acuerdo con los principios de la medicina basada en la evidencia, utilizamos la estructura PICO (Paciente-Intervención-Comparación-Outcome/resultados [Guyatt et al., 2008; Oxman, Schünemann y Fretheim, 2006]) para formular la siguiente pregunta de revisión: «¿Cuál es el efecto de una intervención farmacológica y/o psicológica para el tratamiento de pacientes adultos con un trastorno de ansiedad y un TUS?», entendiendo a pacientes como: pacientes adultos con trastorno de ansiedad y trastorno por consumo de sustancias/alcohol/cannabis/cocaína/nicotina coexistente/ concurrente; intervenciones: cualquier tratamiento farmacológico O psicológico; comparador: placebo $\mathrm{O}$ cualquier tratamiento farmacológico $\mathrm{O}$ cualquier tratamiento psicológico y resultados: reducción de los síntomas clínicos del trastorno de ansiedad; mejora del trastorno por consumo de sustancias y mejora de las medidas pragmáticas y funcionales. La población objetivo de esta guía clínica fueron pacientes mayores de 18 años diagnosticados con AD y TUS (incluyendo cannabis, cocaína, alcohol y/o nicotina). No se incluyó el trastorno por consumo de opioides porque no se encontraron revisiones sistemáticas con o sin metaanálisis o ensayos clínicos aleatorizados.

\section{Búsqueda bibliográfica}

Realizamos una búsqueda exhaustiva de la literatura en MEDLINE, PsycINFO, Embase, Scopus, Web of Science, Cochrane Library y Pubmed hasta mayo de 2018. Se utilizaron los siguientes términos de búsqueda:

- Pubmed (pharmacological intervention)

( ( (“Stress Disorders, Post-Traumatic"[Mesh] OR "Obsessive-Compulsive Disorder"[Mesh] OR "Panic Disorder"[Mesh] OR "Anxiety Disorders"[Mesh] OR posttraumatic stress disorder* OR obsessive compulsive disorder* OR panic disorder* OR anxiety disorder*)) AND (substance abuse OR substance dependence OR substance use OR comorbidity OR misuse OR co-occurr* OR coexist* OR concurren* OR dual diagnosis OR dual disorder OR dual pathology OR "Diagnosis, Dual (Psychiatry)"[Mesh])) AND ("Alcohol Drinking"[Mesh] OR
"Drinking Behavior" [Mesh] OR "alcohol use" OR "alcohol abuse" OR "nicotine use" OR "Marijuana Abuse"[Mesh] OR "Marijuana Smoking”[Mesh] OR "cannabis use" OR "Cocaine-Related Disorders"[Mesh] OR "cocaine use" OR "cocaine abuse")) AND (varenicline OR "varenicline"[Supplementary Concept] OR nicotine replacement therapy OR "Bupropion"[Mesh] OR bupropion OR "topiramate"[Supplementary Concept] OR topiramate OR "acamprosate"[Supplementary Concept] OR acamprosate OR "Naltrexone"[Mesh] OR naltrexone OR anticraving OR "Cyanamide"[Mesh] OR cyanamide OR "Disulfiram"[Mesh] OR disulfiram OR antidipsotropic OR "Antipsychotic Agents"[Mesh] OR antipsychotics OR "Benzodiazepines"[Mesh] OR benzodiazepines OR lamotrigine OR "lamotrigine"[Supplementary Concept] OR valproate OR "Valproic Acid"[Mesh] OR divalproex OR "Lithium"[Mesh] OR lithium OR "Serotonin Uptake Inhibitors"[Mesh] OR "Serotonin Uptake Inhibitors"[Pharmacological Action] OR "duloxetine"[Supplementary Concept] OR duloxetine OR ssris OR "Antidepressive Agents, Tricyclic"[Mesh] OR “Antidepressive Agents, Tricyclic"[Pharmacological Action] OR tricyclic antidepressant OR nsri).

- Límites: Review, Systematic Reviews, Meta-Analysis, Clinical Trial; Young Adult: 19-44 years; Middle Aged: 45-64 years.

( ( (“Stress Disorders, Post-Traumatic"[Mesh] OR “Obsessive-Compulsive Disorder"[Mesh] OR "Panic Disorder"[Mesh] OR "Anxiety Disorders"[Mesh] OR posttraumatic stress disorder* OR obsessive compulsive disorder* OR panic disorder* OR anxiety disorder*)) AND (substance abuse OR substance dependence OR substance use OR comorbidity OR misuse OR co-occurr* OR coexist* OR concurren* OR dual diagnosis OR dual disorder OR dual pathology OR "Diagnosis, Dual (Psychiatry)"[Mesh])) AND ("Alcohol Drinking"[Mesh] OR "Drinking Behavior" [Mesh] OR "alcohol use" OR "alcohol abuse" OR "nicotine use" OR "Marijuana Abuse"[Mesh] OR "Marijuana Smoking" [Mesh] OR "cannabis use" OR "Cocaine-Related Disorders" [Mesh] OR "cocaine use" OR "cocaine abuse")) AND ("Lithium"[Mesh] OR "Lithium Chloride"[Mesh] OR "Lithium Carbonate"[Mesh] OR lithium OR "Valproic Acid" [Mesh] OR valproate OR "lamotrigine 2-N-glucuronide"[Supplementary Concept] OR lamotrigine OR carbamazepine OR oxcarbazepine OR mood stabilizer*).

- Límites: Review, Systematic Reviews, Meta-Analysis, Clinical Trial; Young Adult: 19-44 years; Middle Aged: 45-64 years.

( ( (“Stress Disorders, Post-Traumatic”[Mesh] OR "Obsessive-Compulsive Disorder"[Mesh] OR "Panic Disorder" [Mesh] OR “Anxiety Disorders"[Mesh] OR posttraumatic stress disorder* OR obsessive compulsive disorder* OR panic disorder* OR anxiety disorder*)) AND (substance abuse OR substance dependence OR substance use 
OR comorbidity OR misuse OR co-occurr* OR coexist* OR concurren* OR dual diagnosis OR dual disorder OR dual pathology OR "Diagnosis, Dual (Psychiatry)" [Mesh])) AND ("Alcohol Drinking"[Mesh] OR "Drinking Behavior" [Mesh] OR "alcohol use" OR "alcohol abuse" OR "nicotine use" OR "Marijuana Abuse"[Mesh] OR "Marijuana Smoking” [Mesh] OR "cannabis use" OR "Cocaine-Related Disorders" [Mesh] OR "cocaine use" OR "cocaine abuse”)) AND ("Benzodiazepines"[Mesh] OR benzodiazepines).

- Límites: Review, Systematic Reviews, Meta-Analysis, Clinical Trial; Young Adult: 19-44 years; Middle Aged: 45-64 years.

( ( (“Stress Disorders, Post-Traumatic"[Mesh] OR "Obsessive-Compulsive Disorder"[Mesh] OR "Panic Disorder"[Mesh] OR "Anxiety Disorders"[Mesh] OR posttraumatic stress disorder* OR obsessive compulsive disorder* OR panic disorder* OR anxiety disorder*)) AND (substance abuse OR substance dependence OR substance use OR comorbidity OR misuse OR co-occurr* OR coexist* OR concurren* OR dual diagnosis OR dual disorder OR dual pathology OR "Diagnosis, Dual (Psychiatry)"[Mesh])) AND ("Alcohol Drinking"[Mesh] OR "Drinking Behavior" [Mesh] OR "alcohol use" OR "alcohol abuse" OR "nicotine use" OR "Marijuana Abuse"[Mesh] OR "Marijuana Smoking" [Mesh] OR "cannabis use" OR "Cocaine-Related Disorders" [Mesh] OR "cocaine use" OR "cocaine abuse”)) AND ("Antipsychotic Agents"[Mesh] OR antipsychotic*).

- Límites: Review, Systematic Reviews, Meta-Analysis, Clinical Trial; Young Adult: 19-44 years; Middle Aged: 45-64 years.

( ( ( “Stress Disorders, Post-Traumatic"[Mesh] OR "Obsessive-Compulsive Disorder"[Mesh] OR "Panic Disorder"[Mesh] OR “Anxiety Disorders"[Mesh] OR posttraumatic stress disorder* OR obsessive compulsive disorder* OR panic disorder* OR anxiety disorder*)) AND (substance abuse OR substance dependence OR substance use OR comorbidity OR misuse OR co-occurr* OR coexist* OR concurren* OR dual diagnosis OR dual disorder OR dual pathology OR "Diagnosis, Dual (Psychiatry)"[Mesh])) AND ("Alcohol Drinking"[Mesh] OR "Drinking Behavior" [Mesh] OR "alcohol use" OR "alcohol abuse" OR "nicotine use" OR "Marijuana Abuse"[Mesh] OR "Marijuana Smoking"[Mesh] OR "cannabis use" OR "Cocaine-Related Disorders" [Mesh] OR "cocaine use" OR "cocaine abuse")) AND (varenicline OR "varenicline" [Supplementary Concept] OR nicotine replacement therapy OR "Bupropion"[Mesh] OR bupropion OR "topiramate"[Supplementary Concept] OR topiramate OR "acamprosate"[Supplementary Concept] OR acamprosate OR "Naltrexone"[Mesh] OR naltrexone OR anticraving OR "Cyanamide"[Mesh] OR cyanamide OR "Disulfiram"[Mesh] OR disulfiram OR antidipsotropic).
- Límites: Review, Systematic Reviews, Meta-Analysis, Clinical Trial; Young Adult: 19-44 years; Middle Aged: 45-64 years.

- Pubmed (psychological intervention).

( ( ( Stress Disorders, Post-Traumatic[Mesh] OR "Obsessive-Compulsive Disorder"[Mesh] OR "Panic Disorder" [Mesh] OR “Anxiety Disorders"[Mesh] OR posttraumatic stress disorder* OR obsessive compulsive disorder* OR panic disorder* OR anxiety disorder*)) AND (substance abuse OR substance dependence OR substance use OR comorbidity OR misuse OR co-occurr* OR coexist* OR concurren* OR dual diagnosis OR dual disorder OR dual pathology OR "Diagnosis, Dual (Psychiatry)"[Mesh])) AND ("Alcohol Drinking"[Mesh] OR "Drinking Behavior" [Mesh] OR "alcohol use" OR "alcohol abuse" OR "nicotine use" OR "Marijuana Abuse"[Mesh] OR "Marijuana Smoking” [Mesh] OR "cannabis use" OR "Cocaine-Related Disorders" [Mesh] OR "cocaine use" OR "cocaine abuse”)) AND ("behavioral therapy" Or therapy OR "cognitive therapy” OR "social skills” OR "contingency management” OR "time out” OR "reinforcement programs" OR "token economy” OR self-help OR "motivational interview" OR mindfulness OR "cue exposure" OR self-control OR psychoeducation OR psychotherapy).

- Límites: Review, Systematic Reviews, Meta-Analysis, Clinical Trial; Young Adult: 19-44 years; Middle Aged: 45-64 years.

- Pubmed (exhaustive)

( ((Stress Disorders, Post-Traumatic[Mesh] OR "Obsessive-Compulsive Disorder"[Mesh] OR "Panic Disorder"[Mesh] OR “Anxiety Disorders"[Mesh] OR posttraumatic stress disorder* OR obsessive compulsive disorder* OR panic disorder* OR anxiety disorder*) AND ("Alcohol Drinking”[Mesh] OR "Drinking Behavior" [Mesh] OR alcohol [Title/Abstract] OR nicotine [Title/Abstract] OR "Marijuana Abuse" [Mesh] OR "Marijuana Smoking”[Mesh] OR marijuana[Title/Abstract] OR "cannabis"[Title/Abstract] OR "Cocaine-Related Disorders" [Mesh] OR cocaine[Title/Abstract] OR "substance abuse"[Title/Abstract] OR "substance dependence"[Title/Abstract] OR "substance use"[Title/Abstract] OR misuse[Title/Abstract] OR dual diagnosis[Title/Abstract] OR "dual disorder"[Title/Abstract] OR "dual pathology"[Title/Abstract] OR "Diagnosis, Dual (Psychiatry)"[Mesh]))).

- Límites: Systematic review, Meta-Analysis; +19 years.

( ( (Stress Disorders, Post-Traumatic[Mesh] OR "Obsessive-Compulsive Disorder"[Mesh] OR "Panic Disorder" [Mesh] OR “Anxiety Disorders"[Mesh] OR posttraumatic stress disorder* OR obsessive compulsive disorder* OR panic disorder* OR anxiety disorder*) AND ("Alcohol Drinking”[Mesh] OR "Drinking Behavior" [Mesh] OR alcohol [Title/Abstract] OR nicotine [Title/Abstract] OR "Marijuana Abuse"[Mesh] OR "Marijuana Smoking"[Mesh] OR marijuana[Title/Abstract] OR "cannabis"[Title/Abstract] 
OR “Cocaine-Related Disorders" [Mesh] OR cocaine[Title/Abstract] OR "substance abuse"[Title/Abstract] OR "substance dependence"[Title/Abstract] OR "substance use"[Title/Abstract] OR misuse[Title/Abstract] OR dual diagnosis[Title/Abstract] OR "dual disorder"[Title/ Abstract] OR “dual pathology"[Title/Abstract] OR "Diagnosis, Dual (Psychiatry)"[Mesh]))) AND (Meta-Analysis[ptyp] OR systematic[sb] OR meta-analysis[ti] OR metaanalysis [ti] OR systematic review [ti]).

( ( Stress Disorders, Post-Traumatic[Mesh] OR "Obsessive-Compulsive Disorder"[Mesh] OR "Panic Disorder"[Mesh] OR "Anxiety Disorders"[Mesh] OR posttraumatic stress disorder* OR obsessive compulsive disorder* OR panic disorder* OR anxiety disorder*) AND ("Alcohol Drinking" [Mesh] OR "Drinking Behavior" [Mesh] OR alcohol [Title/Abstract] OR nicotine [Title/Abstract] OR "Marijuana Abuse"[Mesh] OR "Marijuana Smoking”[Mesh] OR marijuana[Title/Abstract] OR "cannabis"[Title/Abstract] OR "Cocaine-Related Disorders" [Mesh] OR cocaine[Title/Abstract] OR "substance abuse"[Title/Abstract] OR "substance dependence"[Title/Abstract] OR "substance use"[Title/Abstract] OR misuse[Title/Abstract] OR dual diagnosis[Title/Abstract] OR "dual disorder"[Title/Abstract] OR "dual pathology"[Title/Abstract] OR "Diagnosis, Dual (Psychiatry)"[Mesh]))).

- Límites: Randomized Controlled Trial; +19 years.

\section{Evaluación de la calidad de la evidencia y formulación de las recomendaciones}

Dada la amplia variación en la metodología de los estudios, los resultados informados y el número limitado de informes de investigación originales que se centraron en cada tratamiento farmacológico o psicológico, basamos este informe en un resumen cualitativo de toda la evidencia disponible. La evaluación de la calidad de la evidencia para cada pregunta PICO se realizó siguiendo las recomendaciones del grupo de trabajo GRADE (Grading of Recommendations Assessment, Development and Evaluation) (www.gradeworkinggroup.org) (Guyatt et al., 2008; Guyatt et al., 2011; Mustafa et al., 2013). Cada artículo se leyó en detalle y se evaluó críticamente de acuerdo con GRADE, luego se discutió entre los autores, lo que resultó en una puntuación general de evaluación de calidad, posteriormente revisada por resultado individual. Los factores que redujeron la calidad de la evidencia fueron el diseño del estudio, inconsistencia, sesgo de publicación, precisión y direccionalidad, mientras que los factores que aumentaron la calidad de la evidencia fueron la gran magnitud del efecto y el gradiente dosis-respuesta. Las tablas de Resumen de los hallazgos correspondientes a cada PICO están disponibles a petición del interesado.

Todo el proceso culminó en una recomendación clínica que se calificó según su fuerza, con el fin de reflejar el grado de confianza en que los efectos deseables de la adherencia a una recomendación superan los efectos indeseables. Las recomendaciones en el presente documento se formularon de acuerdo con la calidad de la evidencia y el balance entre beneficios y efectos adversos. No fueron incluidos los valores y las preferencias de los pacientes y los análisis de costes. En aras de la claridad, las recomendaciones se dividen aquí por sustancia.

\section{Revisión y evaluación externas}

La revisión y evaluación externas fueron realizadas por un grupo independiente de expertos utilizando el instrumento AGREE II (Appraisal of Guidelines for Research and Evaluation) (Gopalakrishna, Langendam, Scholten, Bossuyt y Leeflang, 2013) (www.agreecollaboration.org). El instrumento AGREE II consta de 23 ítems incluidos en seis dominios evaluables: alcance y propósito, participación de las partes interesadas, rigor de desarrollo, claridad y presentación, aplicabilidad e independencia editorial. Los comentarios planteados por los revisores se incluyeron en el estudio revisado.

Se puede encontrar información más detallada sobre la metodología en publicaciones anteriores (San y Arranz, 2016).

\section{Resultados}

\section{Selección de estudios}

La Figura 1 describe el diagrama de flujo PRISMA que resulta en la selección del estudio. La búsqueda produjo 204 estudios. Se consideraron elegibles para una evaluación adicional 39 estudios. La selección definitiva incluyó 13 estudios. No se incluyeron en la Guía los ensayos abiertos, los estudios de cohortes o de casos y controles, los estudios transversales y observacionales, los informes de casos, las cartas, los carteles y los resúmenes de presentaciones en reuniones y conferencias de especialistas. Solo se incluyeron artículos publicados en inglés. Los datos se extrajeron de los estudios incluidos mediante una plantilla predefinida y la calidad de cada estudio se evaluó mediante criterios estándar. La Tabla 1 presenta un informe resumido de estos estudios.

Pregunta 1 de PICO. ¿Es eficaz la administración de antidepresivos inhibidores selectivos de la recaptación de serotonina (los ISRS) para disminuir los sintomas de la ansiedad en pacientes con TEPT y trastorno por consumo de alcohol concurrente? y ¿es eficaz la administración de antidepresivos inhibidores selectivos de la recaptación de serotonina (los ISRS) para reducir el consumo de alcohol en pacientes con TEPT y trastorno por consumo de alcohol concurrente?

Tres ECA evaluaron el efecto de sertralina más terapia cognitivo-conductual (TCG) versus placebo durante 12 semanas en pacientes con TEPT y consumo de alcohol (Back, Brady, Sonne y Verduin, 2006; Brady, Sonne y Ro- 


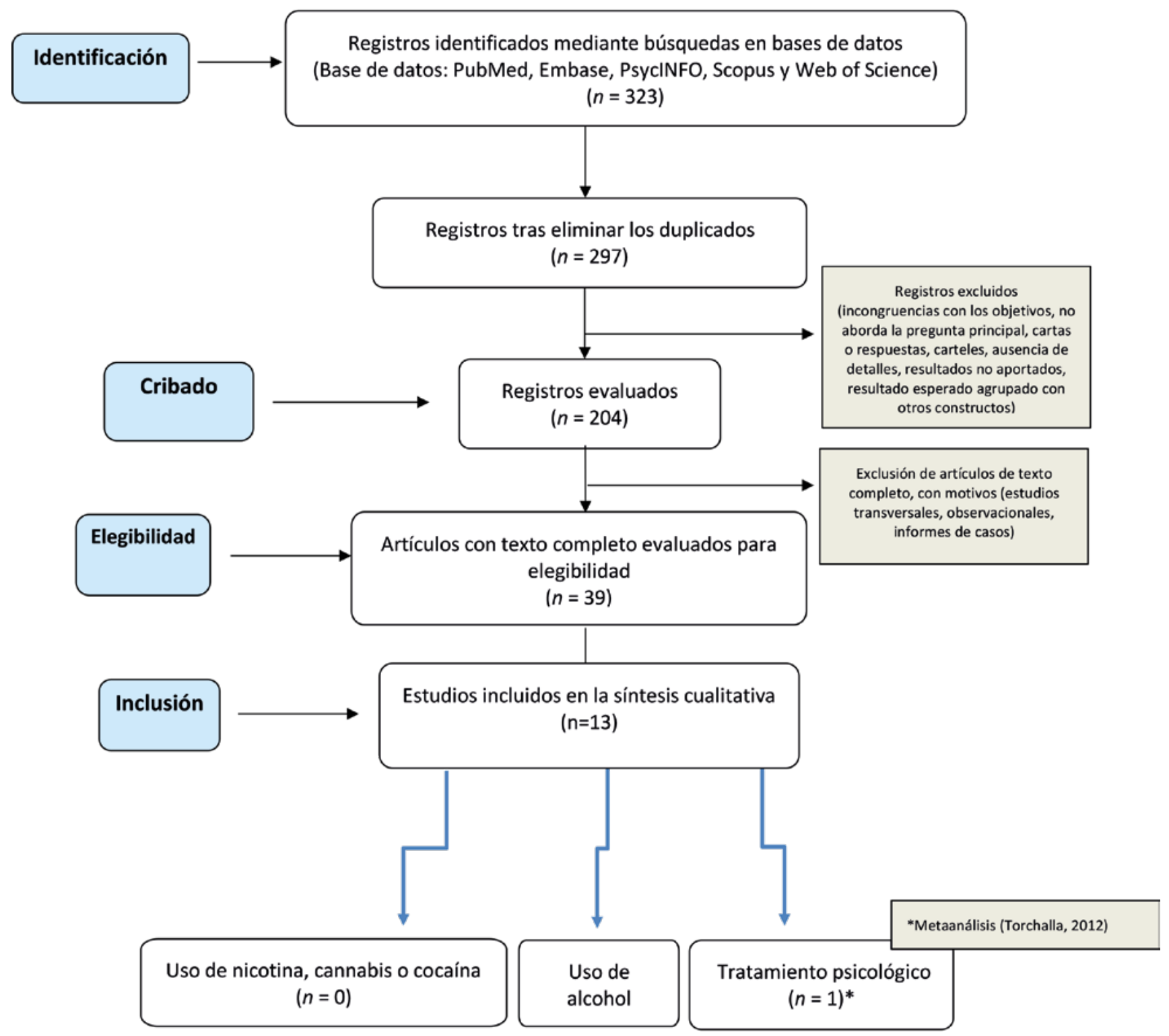

Figura 1. Diagrama de flujo del proceso de selección de estudios.

berts, 1995; Labbate, Sonne, Randal, Anton y Brady, 2004), siendo dos de ellos el mismo estudio (Back et al., 2006; Labbate et al., 2004). Ninguno de los estudios describió la generación de secuencia aleatoria, asignación oculta o protocolo de estudio. El número y el número medio de bebidas por día, así como el número de días de consumo excesivo de alcohol, fueron mayores en el grupo de sertralina (evidencia de calidad moderada) (Brady et al., 1995).

Un ECA realizado en 94 pacientes que cumplían los criterios de TEPT y comorbilidad de dependencia de alcohol evaluó la paroxetina y la gestión clínica/mejora del cumplimiento terapéutico frente a desipramina y la gestión clínica/mejora del cumplimiento terapéutico con o sin naltrexona en los dos brazos, para medir la gravedad del TEPT como principal resultado (Petrakis et al., 2012). La severidad de los síntomas de TEPT se evaluó con la Es- cala para el TEPT Administrada por el Clínico (CAPS) y el tratamiento tuvo una duración de 12 semanas. En la puntuación total CAPS y las subescalas CAPS de re-experiencia, evitación e hiperactivación, las puntuaciones medias fueron más altas en el grupo de paroxetina (evidencia de calidad moderada).

\section{- Recomendaciones}

- En los pacientes con TEPT y trastorno por consumo de alcohol concurrente, no se puede recomendar el uso de antidepresivos ISRS para aliviar los síntomas de ansiedad o reducir el consumo de alcohol (recomendación débil).

Pregunta 2 de PICO. ¿Es eficaz la administración de desipramina para disminuir los síntomas de la ansiedad en pacientes con TEPT y trastorno por consumo de alcohol concurrente? y ¿es eficaz 
Pilar A. Sáiz, Gerardo Flórez, Manuel Arrojo, Miquel Bernardo, Ana González-Pinto, José Manuel Goikolea, Iñaki Zorrilla, Ruth Cunill, Xavier Castells, Elisardo Becoña, Ana López, Marta Torrens, Francina Fonseca, Judit Tirado-Muñoz, Belén Arranz, Marina Garriga, Luis San

Tabla 1. Trastorno de ansiedad y trastorno por uso de alcohol.

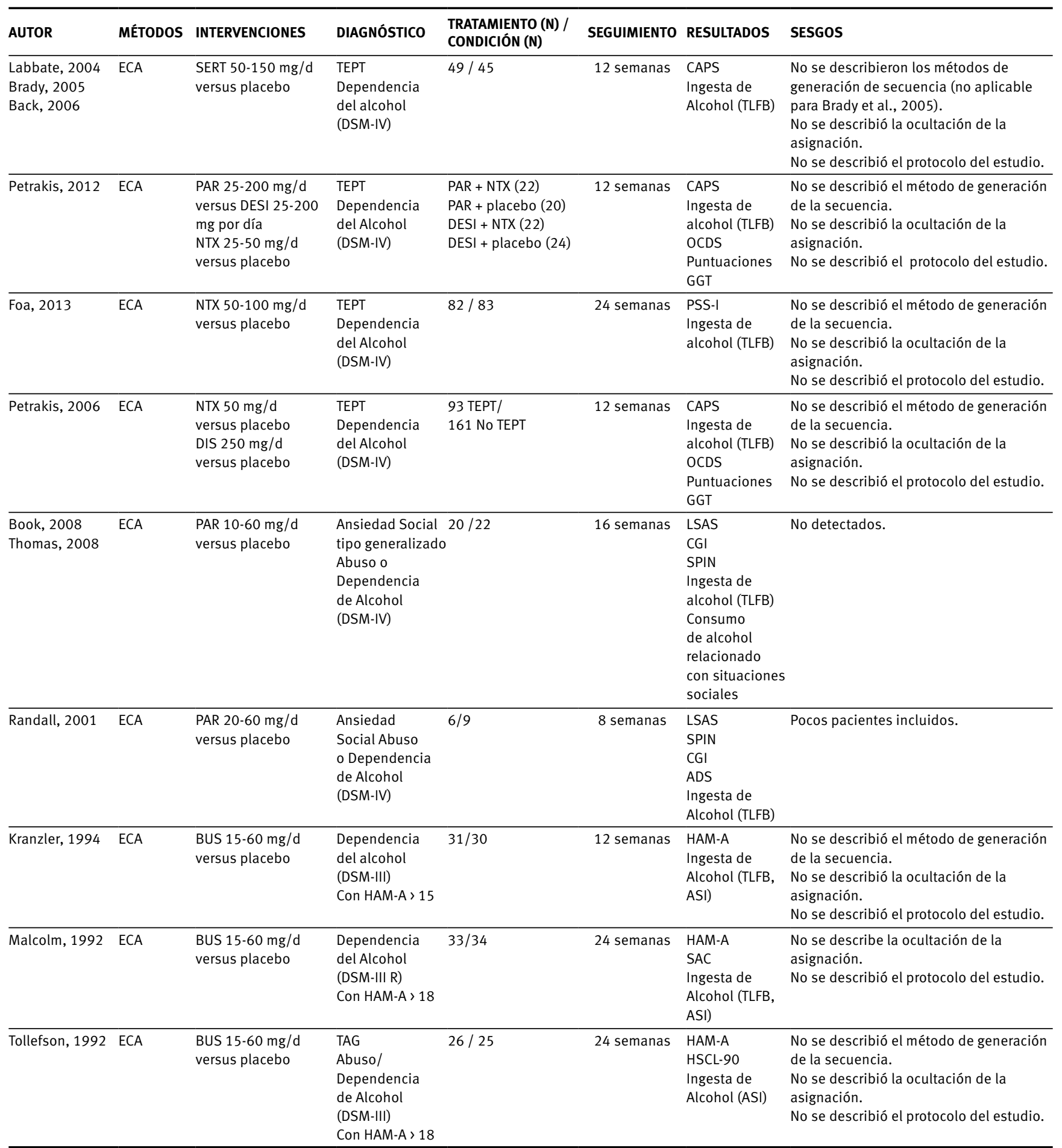

ADS: Alcohol Dependence Scale; ASI: Addiction Severity Index; BUS: Buspirona; CAPS: Clinician administered PTSD Scale; CGI: Clinical Global Impression; DESI: Desipramina; ECA: Ensayo Controlado Aleatorizado; GGT: Gamma-glutamil transpeptidasa; HAM-A: Hamilton Anxiety Rating Scale; HSCL-9o: The Hopkins symptom checklist; ICG: Clinical Global Impressions; LSAS: Liebowitz Social Anxiety Scale; NTX: Naltrexona; OCDS: Obsessive Compulsive Drinking Scale; PAR: Paroxetina; PSS-I: PTSD Symptom Scale- Interview; SPIN: Social Phobia Inventory; PTSD Symptom Scale-Interview; SAC: State Anxiety Scale; SERT: Sertralina; TAG: Trastorno Ansiedad Generalizada; TEPT: Trastorno por Estrés Postraumático; TLFB: Time line follow back.

la administración de desipramina para reducir el consumo de alcohol en pacientes con TEPT y trastorno por consumo de alcohol concurrente?

En el mismo ECA descrito en la pregunta 1 de PICO, se evaluó el efecto de desipramina vs paroxetina para aliviar los síntomas de ansiedad en 94 pacientes que cumplían los criterios para TEPT y trastorno por consumo de alcohol concurrente (Petrakis et al., 2012). Los pacientes tratados con paroxetina, comparado con aquellos tratados con desipramina, obtuvieron puntuaciones 3,82 veces en la puntuación CAPS total, 0,94 veces más alta en la subescala CAPS de re-experiencia, 1,6 veces más alta en la subescala CAPS 
de evitación y 3,82 veces más alta en la subescala CAPS de hiperactivación (evidencia de calidad moderada).

\section{- Recomendaciones}

- En los pacientes con TEPT y trastorno por consumo de alcohol concurrente, se puede recomendar el uso de desipramina sobre los ISRS (paroxetina) para aliviar los síntomas de ansiedad (recomendación débil).

- No se puede recomendar la desipramina en lugar de los ISRS (paroxetina) para reducir el consumo de alcohol (recomendación débil).

Pregunta 3 de PICO. ¿Es eficaz la administración de naltrexona para disminuir los síntomas de la ansiedad en pacientes con TEPT y trastorno por consumo de alcohol concurrente? y ies eficaz la administración de naltrexona para reducir el consumo de alcohol en pacientes con TEPT y trastorno por consumo de alcohol concurrente?

Dos ECA llevados a cabo por el mismo grupo (Petrakis et al., 2006) (Petrakis et al., 2012) evaluaron el tratamiento de 12 semanas con naltrexona frente a placebo asociado con la gestión clínica/mejora del cumplimiento terapéutico en ambos brazos para aliviar los síntomas de ansiedad (cuestionario CAPS) y reducir el consumo de alcohol. Los pacientes tratados con naltrexona obtuvieron puntuaciones más bajas en la puntuación CAPS total y en las subescalas CAPS de re-experiencia, evitación e hiperactivación (evidencia de calidad moderada). En las medidas de consumo de alcohol, los resultados fueron más favorables en el grupo de naltrexona frente a placebo: 19,9 veces más altas para el número medio máximo de días de abstinencia, 4,4 veces más altas para el porcentaje de días de abstinencia, 4,2 veces más bajas para los días de consumo excesivo de alcohol, 6 veces más altas para la abstinencia media durante el período de estudio, y puntuaciones 1,3 veces más bajas en la puntuación total de la Escala de componentes obsesivo-compulsivos de la bebida (OCDS) (evidencia de calidad moderada). Los niveles de gamma-glutamil transferasa (GGT) fueron 3,5 veces más altos en el grupo de naltrexona (evidencia de calidad moderada).

\section{- Recomendaciones}

- En los pacientes con TEPT y trastorno por consumo de alcohol concurrente, se puede recomendar el uso de naltrexona para reducir los síntomas de la ansiedad (recomendación débil).

- No se puede recomendar la naltrexona para reducir el consumo de alcohol (recomendación débil).

Pregunta 4 de PICO. ¿Es eficaz la administración de disulfiram para disminuir los sintomas de la ansiedad en pacientes con TEPT y trastorno por consumo de alcohol concurrente? y ¿es eficaz la administración de disulfiram para reducir el consumo de alcohol en pacientes con TEPT y trastorno por consumo de alcohol concurrente?
Un ECA con 93 pacientes comparó el tratamiento de 12 semanas con naltrexona versus placebo y disulfiram versus placebo más la gestión clínica/mejora del cumplimiento terapéutico (Petrakis et al., 2006). Las puntuaciones totales CAPS y las puntuaciones en las subescalas CAPS fueron más altas en el grupo de disulfiram que en el grupo de placebo (evidencia de calidad moderada). Respecto del consumo de alcohol, el número medio máximo de días consecutivos de abstinencia fue 8 veces más alto, el porcentaje de días de consumo excesivo fue 7,4 más bajo, la abstinencia media durante el período de estudio fue 11 veces más alto, la puntuación total media en el cuestionario OCDS fue 3,8 más baja y la los niveles medios de GGT fueron 24,5 veces más altos en el grupo de disulfiram en comparación con el placebo (evidencia de calidad moderada).

\section{- Recomendaciones}

- En pacientes con TEPT y trastorno por consumo de alcohol concurrente, no se puede recomendar el uso de disulfiram versus placebo para reducir los síntomas de la ansiedad (recomendación débil).

- No se puede recomendar disulfiram frente a placebo para reducir el consumo de alcohol (recomendación débil).

Pregunta 5 de PICO. ¿Es eficaz la administración de naltrexona más disulfiram para disminuir los sintomas de la ansiedad en pacientes con TEPT y trastorno por consumo de alcohol concurrente? y ¿ es eficaz la administración de naltrexona más disulfiram para reducir el consumo de alcohol en pacientes con TEPT y trastorno por consumo de alcohol concurrente?

En el ECA de Petrakis et al. (Petrakis et al., 2006) de 93 pacientes con TEPT y trastorno por consumo de alcohol concurrente en tratamiento durante 12 semanas, uno de los grupos de estudio era naltrexona y disulfiram, con placebo como el comparador. Las puntuaciones totales CAPS y las puntuaciones en las subescalas CAPS fueron más altas en el grupo de naltrexona y disulfiram que en el grupo de placebo (evidencia de calidad moderada). Respecto del consumo de alcohol, el número medio máximo de días consecutivos de abstinencia fue 18,5 veces más alto, el porcentaje de días de abstinencia fue 8,1 veces más alto, el porcentaje de días de consumo excesivo fue 7,9 veces más bajo, la abstinencia media durante el período de estudio fue 6 veces más alta, la puntuación total media en el cuestionario OCDS fue 4,2 veces más baja y los niveles medios de GGT fueron 10,9 veces más altos en el grupo de naltrexona y disulfiram en comparación con el placebo (evidencia de calidad moderada).

\section{- Recomendaciones}

- En pacientes con TEPT y trastorno por consumo de alcohol concurrente, no se puede recomendar el uso de naltrexona y disulfiram versus placebo para reducir los síntomas de la ansiedad (recomendación débil). 
- No se puede recomendar naltrexona más disulfiram frente a placebo para reducir el consumo de alcohol (recomendación débil).

Pregunta 6 de PICO. ¿Es eficaz la administración de antidepresivos inhibidores selectivos de la recaptación de serotonina (los ISRS) para disminuir los sintomas de la ansiedad en pacientes con trastorno de ansiedad social y trastorno por consumo de alcohol concurrente? y ¿ es eficaz la administración de antidepresivos inhibidores selectivos de la recaptación de serotonina (los ISRS) para reducir el consumo de alcohol en pacientes con trastorno de ansiedad social y trastorno por consumo de alcohol concurrente?

Tres ECA abordaron la comparación de paroxetina versus placebo en pacientes con trastorno de ansiedad social y trastorno por consumo de alcohol concurrente (Book, Thomas, Randall y Randall, 2008; Randall et al., 2001; Thomas, Randall, Book y Randall, 2008), pero en dos de ellos (Book et al., 2008; Randall et al., 2001) no fueron analizados los resultados de interés. En un ECA de 16 semanas con 93 pacientes (Thomas et al., 2008), no había ninguna diferencia entre paroxetina y placebo en bebidas por día de consumo de alcohol, porcentaje de días de abstinencia o porcentaje de días de consumo excesivo (evidencia de calidad moderada). Sin embargo, el porcentaje de consumo de alcohol previo a situaciones sociales fue 24 veces más bajo y durante situaciones sociales fue 13 veces más bajo en el grupo de paroxetina (evidencia de calidad moderada).

\section{- Recomendaciones}

- En pacientes con trastorno de ansiedad social y trastorno por consumo de alcohol concurrente, no se pueden recomendar antidepresivos ISRS frente a placebo para reducir los síntomas de la ansiedad (recomendación débil).

- Se pueden recomendar antidepresivos ISRS frente a placebo para reducir el consumo de alcohol (recomendación débil). Esta recomendación asigna un valor relativamente alto a la reducción del consumo de alcohol relacionado con la ansiedad social, y un valor relativamente bajo a la reducción del consumo de alcohol en general.

Pregunta 7 de PICO. ¿Es eficaz la administración de buspirona para disminuir los sintomas de la ansiedad en pacientes con un trastorno de ansiedad y trastorno por consumo de alcohol concurrente? y ¿̇es eficaz la administración de buspirona para reducir el consumo de alcohol en pacientes con trastorno por ansiedad y trastorno por consumo de alcohol concurrente?

Una ECA evaluó el tratamiento con buspirona vs placebo durante 12 semanas en 61 pacientes con un trastorno de ansiedad y trastorno por consumo de alcohol concurrente (Kranzler et al., 1994). Las puntuaciones medias en el cuestionario HAM-A eran 1,5 más bajas en el grupo de buspirona que en el grupo de placebo (evidencia de calidad moderada). Además, la media de días de consumo de alcohol fue 6 veces más baja, bebidas por día de consumo de alcohol fue 3,9 veces más baja y el número de bebidas por día de consumo de alcohol fue 4,6 veces más baja en el grupo de buspirona versus placebo (evidencia de calidad moderada).

\section{- Recomendaciones}

- En los pacientes con un trastorno de ansiedad y trastorno por consumo de alcohol concurrente, no se puede recomendar el uso de buspirona para aliviar los síntomas de ansiedad o reducir el consumo de alcohol (recomendación débil).

Pregunta 8 de PICO. ¿Es eficaz el tratamiento psicológico para reducir los sintomas del trastorno por estrés postraumático (TEPT) o para reducir el consumo de drogas de abuso en pacientes con TEPT y TUS?

Un metaanálisis de nueve ECA evaluó el impacto de programas de tratamiento con psicoterapia integrada vs no integrada en los pacientes con TEPT y TUS concurrente (Hesse, 2009; Torchalla, Nosen, Rostam y Allen, 2012). La mayoría de las intervenciones incluyeron una combinación de apoyo social, psicoeducación y TCC, capacitación en resolución de problemas, habilidades de regulación interpersonal y emocional, desarrollo de estrategias de afrontamiento ante estímulos relacionados con traumas y sustancias y programas integrados para el cese del tabaquismo. No hubo diferencias significativas entre los programas de tratamiento integrado y comparadores para el cambio de síntomas de TEPT o la mejora del TUS (evidencia de muy baja calidad).

\section{- Recomendaciones}

- En pacientes con TEPT y TUS concurrente, no se puede hacer ninguna recomendación con respecto a la intervención psicológica más adecuada para mejorar los síntomas del TEPT y reducir el uso de sustancias.

\section{Discusión}

Esta revisión resume las intervenciones farmacológicos y psicosociales que han sido llevadas a cabo en trastornos de ansiedad con un diagnóstico comórbido, incluyendo ansiedad social, trastorno de pánico, agorafobia, fobia simple, fobia social, trastorno de ansiedad generalizada, trastorno obsesivo-compulsivo y TEPT y TUS, y además proporciona recomendaciones clínicas respecto de cuáles elementos de intervención son útiles para hacer frente a los síntomas del uso de sustancias versus los síntomas de ansiedad en pacientes con estas afecciones concurrentes.

La escasez de estudios aleatorizados en personas con trastornos de ansiedad y comorbilidad de un TUS sigue siendo preocupante, dada la enorme carga que suponen. Muy pocos de los ensayos aleatorizados realizados hasta la fecha han proporcionado evidencia consistente para el manejo tanto 
de la ansiedad como del uso de sustancias. Hay ensayos clínicos disponibles únicamente para TEPT y para trastorno de ansiedad social. En cuanto al diagnóstico comórbido de trastorno por uso de sustancias, todos los estudios han incluido pacientes con consumo de alcohol; ninguno de ellos ha abordado el consumo de cocaína, cannabis o nicotina. Aunque algunos tratamientos han mostrado beneficios para los síntomas de ansiedad sin ningún beneficio para el consumo de alcohol u otras sustancias, solo se han ensayado enfoques farmacológicos limitados (sertralina, desipramina, paroxetina, buspirona, naltrexona y disulfiram).

Nuestros resultados sugieren que 1) podemos (débilmente) recomendar el uso de desipramina sobre la paroxetina para aliviar los síntomas de ansiedad en pacientes con un TEPT y consumo de alcohol; 2) en estos pacientes, el uso de naltrexona para reducir los síntomas de ansiedad es también recomendable (fuerza débil); y 3) se pueden recomendar antidepresivos ISRS frente a placebo para reducir el consumo de alcohol (recomendación débil). Esta recomendación asigna un valor relativamente alto a la reducción del consumo de alcohol relacionado con la ansiedad social, y un valor relativamente bajo a la reducción del consumo de alcohol en general.

Durante el transcurso de las presentes recomendaciones, Gimeno et al. (2017) reportaron una revisión narrativa de la evidencia científica y recomendaciones para el tratamiento de pacientes con dependencia al alcohol y un trastorno de ansiedad. Sus recomendaciones no están de acuerdo con las nuestras, debido a varias diferencias metodológicas en ambos estudios. En nuestro estudio, la calidad de la evidencia se calificó siguiendo el sistema GRADE, que es un procedimiento más estructurado y rígido, y se evaluó mediante el instrumento AGREE II. En segundo lugar, Gimeno et al. (2017), incluyeron algunos estudios abiertos y retrospectivos, que no fueron incluidos en nuestro estudio. Además, algunas de sus recomendaciones se basaron en estudios realizados sobre depresión mayor con síntomas de ansiedad, o en pacientes con trastorno por consumo de alcohol en los que se puntuaron los síntomas de ansiedad (no trastorno de ansiedad).

Nuestra revisión señala la necesidad de realizar más investigaciones en esta área y de estudios más grandes, multisitio con muestras generalizables para proporcionar evidencia más definitiva para la práctica clínica. Esta investigación debe garantizar una aleatorización adecuada, el uso de un comparador activo y seguimientos a largo plazo para establecer la sostenibilidad de los resultados del tratamiento.

\section{Reconocimientos}

Este estudio ha recibido apoyo financiero de: Servicio Gallego de Salud, SERGAS; Sociedad Española de Psiquiatría Biológica (SEPB), Pla Director de Salut Mental i Adiccions, Barcelona.

\section{Conflicto de intereses}

Los autores declaran la inexistencia de conflicto de interés alguno en relación a este artículo.

\section{Referencias}

American Psychiatric Association. (2013). Diagnostic and Statistical Manual of Mental Disorders (5th ed.). Washington, DC: American Psychiatric Association. doi:10.1176/appi. books.9780890425596.

Back, S. E., Brady, K. T., Sonne, S. C. y Verduin, M. L. (2006). Symptom improvement in co-occurring PTSD and alcohol dependence. The Journal of Nervous and MentalDisease, 194, 690-696. doi:10.1097/01.nmd.0000235794.12794.8a.

Book, S. W., Thomas, S. E., Randall, P. K. y Randall, C. L. (2008). Paroxetine reduces social anxiety in individuals with a co-occurring alcohol use disorder. Journal of Anxiety Disorders, 22, 310-318. doi:10.1016/j.janxdis.2007.03.001.

Brady, K. T., Killeen, T. K., Brewerton, T. y Lucerini, S. (2000). Comorbidity of psychiatric disorders and posttraumatic stress disorder. The Journal of Clinical Psychiatry, 61 (Suppl 7), 22-32.

Brady, K. T., Sonne, S. C. y Roberts, J. M. (1995). Sertraline treatment of comorbid posttraumatic stress disorder and alcohol dependence. Journal of Clinical Psychiatry, 56, 502-505.

Clark, H. W., Masson, C. L., Delucchi, K. L., Hall, S. M. y Sees, K. L. (2001). Violent traumatic events and drug abuse severity. Journal of Substance Abuse Treatment, 20, 121127. doi:10.1016/s0740-5472(00)00156-2.

Compton, W. M., Thomas, Y. F., Stinson, F. S. y Grant, B. F. (2007). Prevalence, correlates, disability, and comorbidity of DSM-IV alcohol abuse and dependence in the United States: Results from the National Epidemiologic Survey on Alcohol and Related Conditions. Archives of General Psychiatry, 64, 830-842. doi:10.1001/archpsyc.64.5.566.

Driessen, M., Schulte, S., Luedecke, C., Schaefer, I., Sutmann, F., Ohlmeier, M.,... Havemann-Reinecke, U. (2008). Trauma and PTSD in patients with alcohol, drug, or dual dependence: A multi-center study. Alcoholism, Clinical and Experimental Research, 32, 481-488. doi:10.1111/ j.1530-0277.2007.00591.x.

Gimeno, C., Dorado, M. L., Roncero, C., Szerman, N., Vega, P., Balanzá-Martínez, V. y Alvarez, F. J. (2017). Treatment of comorbid alcohol dependence and anxiety disorder: Review of the scientific evidence and recommendations for treatment. Frontiers in Psychiatry, 8, 1-14. doi:10.3389/ fpsyt.2017.00173.

Gopalakrishna, G., Langendam, M. W., Scholten, R. J., Bossuyt, P. M. y Leeflang, M. M. (2013). Guidelines for guideline developers: A systematic review of grading systems for medical tests. Implementation Science, 8, 78. doi:10.1186/1748-5908-8-78. 
Guyatt, G. H., Oxman, A. D., Kunz, R., Falck-Ytter, Y., Vist, G. E., Liberati, A. y Schünemann, H. J. (2008). Going from evidence to recommendations. BMJ, 336, 1049-1051. doi:10.1136/bmj.39493.646875.AE.

Guyatt, G. H., Oxman, A. D., Sultan, S., Glasziou, P., Akl, E. A., Alonso-Coello, P.,... Schünemann, H. J. (2011). GRADE guidelines: 9. Rating up the quality of evidence. Journal of Clinical Epidemiology, 64, 1311-1316. doi:10.1016/j. jclinepi.2011.06.004.

Hesse, M. (2009). Integrated psychological treatment for substance use and co-morbid anxiety or depression vs. treatment for substance use alone. A systematic review of the published literature. BMC Psychiatry, 9, 6. doi:10.1186/1471-244X-9-6.

Hunt, G. E., Siegfried, N., Morley, K., Sitharthan, T. y Cleary, M. (2013). Psychosocial interventions for people with both severe mental illness and substance misuse. The Cochrane Database of Systematic Reviews, 10, CD001088. doi:10.1002/14651858.CD001088.pub3.

Ipser, J. C., Wilson, D., Akindipe, T. O., Sager, C. y Stein, D. J. (2015). Pharmacotherapy for anxiety and comorbid alcohol use disorders. Cochrane Database Syst Rev, 1, Cd007505. doi:10.1002/14651858.CD007505.pub2.

Iqbal, M. N., Levin, C. J. y Levin, F. R. (2019). Treatment for substance use disorder with co-occurring mental illness. Focus, 17, 88-97. doi:10.1176/appi.focus.20180042.

Kranzler, H. R., Burleson, J. A., Del Boca, F. K., Babor, T. F., Korner, P., Brown, J. y Bohn, M. J. (1994). Buspirone treatment of anxious alcoholics. A placebo-controlled trial. Archives of General Psychiatry, 51, 720-731.

Labbate, L. A., Sonne, S. C., Randal, C. L., Anton, R. F. y Brady, K. T. (2004). Does comorbid anxiety or depression affect clinical outcomes in patients with post-traumatic stress disorder and alcohol use disorders? Comprehensive Psychiatry, 45, 304-310. doi:10.1016/j.comppsych.2004.03.015.

Lopez-Quintero, C., Perez de los Cobos, J., Hasin, D. S., Okuda, M., Wang, S., Grant, B. F. y Blanco, C. (2011). Probability and predictors of transition from first use to dependence on nicotine, alcohol, cannabis, and cocaine: results of the National Epidemiologic Survey on Alcohol and Related Conditions (NESARC). Drug and Alcohol Dependence, 115, 120-130. doi:10.1016/j.drugalcdep.2010.11.004.

María-Ríos, C. E. y Morrow, J. D. (2020). Mechanisms of shared vulnerability to post-traumatic stress disorder and substance use disorders. Frontiers in Behavioral Neuroscience, 14, 1-21. doi:10.3389/fnbeh.2020.00006.

McCauley, J. L., Killeen, T., Gros, D. F., Brady, K. T. y Back, S. E. (2012). Posttraumatic stress disorder and co-occurring substance use disorders: Advances in assessment and treatment. Clinical Psychology, 19. doi:10.1111/cpsp.12006.

Mustafa, R. A., Santesso, N., Brozek, J., Akl, E. A., Walter, S. D., Norman, G.,... Schünemann, H. J. (2013). The GRADE approach is reproducible in assessing the quali- ty of evidence of quantitative evidence syntheses. Journal of Clinical Epidemiology, 66, 736-742. doi:10.1016/j.jclinepi.2013.02.004.

Oxman, A. D., Schünemann, H. J. y Fretheim, A. (2006). Improving the use of research evidence in guideline development: 2. Priority setting. Health Research Policy and Systems, 4, 14. doi:10.1186/1478-4505-4-14.

Petrakis, I. L., Poling, J., Levinson, C., Nich, C., Carroll, K., Ralevski, E. y Rounsaville, B. (2006). Naltrexone and disulfiram in patients with alcohol dependence and comorbid post-traumatic stress disorder. Biological Psychiatry, 60, 777-783. doi:10.1016/j.biopsych.2006.03.074.

Petrakis, I. L., Ralevski, E., Desai, N., Trevisan, L., Gueorguieva, R., Rounsaville, B. y Krystal, J. H. (2012). Noradrenergic vs serotonergic antidepressant with or without naltrexone for veterans with PTSD and comorbid alcohol dependence. Neuropsychopharmacology, 37, 996-1004. doi:10.1038/npp.2011.283.

Randall, C. L., Johnson, M. R., Thevos, A. K., Sonne, S. C., Thomas, S. E., Willard, S. L.,... Davidson, J. R. (2001). Paroxetine for social anxiety and alcohol use in dual-diagnosed patients. Depression and Anxiety, 14, 255-262. doi:10.1002/da.1077.

Sáiz, P. A., Jimenez, L., Díaz, E. M., García-Portilla, M. P., Marina, P., Al-Halabí, S.,... Ruiz, P. (2014). Patología dual en trastornos de ansiedad: Recomendaciones en el tratamiento farmacológico. Adicciones, 26, 254-274. doi:10.20882/adicciones.7.

San, L. y Arranz, B. (2016). Clinical guideline for the treatment of dual pathology in the adult population. Adicciones, 28, 3-5. doi:10.20882/adicciones. 784.

Smith, J. P. y Book, S. W. (2008). Anxiety and substance use disorders: A review prevalence of comorbid anxiety and substance use disorders. The Psychiatric Times, 25, 19-23.

Thomas, S. E., Randall, P. K., Book, S. W. y Randall, C. L. (2008). A complex relationship between co-occurring social anxiety and alcohol use disorders: What effect does treating social anxiety have on drinking? Alcoholism, Clinical and Experimental Research, 32, 77-84. doi:10.1111/ j.1530-0277.2007.00546.x.

Torchalla, I., Nosen, L., Rostam, H. y Allen, P. (2012). Integrated treatment programs for individuals with concurrent substance use disorders and trauma experiences: A systematic review and meta-analysis. Journal of Substance Abuse Treatment, 42, 65-77. doi:10.1016/j.jsat.2011.09.001.

Vorspan, F., Mehtelli, W., Dupuy, G., Bloch, V. y Lépine, J. P. (2015). Anxiety and substance use disorders: Co-occurrence and clinical issues. Current Psychiatry Reports, 17. doi:10.1007/s11920-014-0544-y. 